\author{
Justyna Duch-Adamczyk \\ ORCID: 0000-0002-4199-0472 \\ Adam-Mickiewicz-Universität, Poznań
}

DOI: $10.19195 / 0435-5865.143 .10$

\title{
Image-Arbeit im Interview - Fallstudie am Beispiel eines „Spiegel“-Interviews mit Boris Becker
}

\begin{abstract}
s
Im vorliegenden Beitrag wird der Versuch unternommen, ein Interview mit einer prominenten Person unter dem Aspekt der Image-Arbeit und der Emotionalität im Gespräch zu untersuchen. Vor diesem Hintergrund werden der Begriff des face und Marker der Emotionalität besprochen. Am Beispiel eines Interviews mit Boris Becker wird gezeigt, wie Images variieren können und wie sie sprachlich manifestiert werden. Anschließend wird die gesamte Analyse ausgewertet und zusammengefasst.
\end{abstract}

Schlüsselwörter: Interview, Image-Arbeit, face

\section{Image-work in interview - illustrated by the example of an interview with Boris Becker, extracted from the magazine "Der Spiegel"}

The paper deals with the language and strategies of self-presentation in an interview with a prominent person. Special attention is given to image-work and emotionality in conversation. Against this background, the concept of the face-work and the emotionality in interviews are discussed. Presented on the example of an interview with Boris Becker, the author attempts to show how images can vary and how they can be expressed verbally. The paper ends with evaluation and conclusions.

Keywords: interview, image-work, face

Justyna Duch-Adamczyk, Uniwersytet im. Adama Mickiewicza, Instytut Lingwistyki Stosowanej, Al. Niepodległości 4, 61-874 Poznań, Polen, E-Mail: jda@amu.edu.pl

Received: 29.09.2017, accepted: 18.05.2018 


\section{Einleitung}

Den Gegenstand meiner Untersuchung stellen Presseinterviews dar, die aufgrund der in ihnen präsenten Mündlich- und Schriftlichkeit ein interessantes Forschungsgebiet bilden. Die Grundlage eines solchen Interviews ist ein Gespräch, das im Nachhinein redaktionell bearbeitet und nachgebessert wird. Wie ein beliebiges Gespräch auch unerwartet verlaufen kann, so nehmen Interviews oft einen überraschenden Verlauf, was sich auf sprachlicher Ebene widerspiegelt.

Presseinterviews sind keine homogene Textsorte. Sie verlaufen sehr verschieden und werden von den Befragten wie den Fragenden für diverse Ziele verwendet. Im Allgemeinen könnte man feststellen, dass beispielsweise Experten in einem Interview eine Sache oder eine Angelegenheit darlegen, für die ein bestimmtes Publikumsinteresse besteht. Etwas anders verhält es sich bei Interviews mit Prominenten, die sich vor allem auf private Anliegen der interviewten Person beziehen. Mein Interesse erweckte ein Interview mit Boris Becker, das im Jahre 2014 in der 34. „Spiegel“-Ausgabe erschien. Bezogen auf dieses Interview möchte ich näher auf die Imagearbeit eingehen und zugleich die sprachliche „Landschaft“ eines Interviews mit Prominenten darstellen.

In meinem Beitrag möchte ich erstens einen Einblick in die face-Untersuchungen geben. Ausgehen werde ich dabei vom face-Begriff und diesen nachfolgend auf die Imagearbeit und Selbstdarstellung im Interview übertragen. Im Weiteren werde ich mich auf Emotionalität im Interview und ihre Marker konzentrieren und das Interview mit Prominenten als Textsorte kurz skizzieren. Anschließend stelle ich das ausgewählte Interview unter dem Aspekt der Selbstdarstellung und Imagearbeit sowie ihrer sprachlichen Manifestation dar.

\section{Zum Begriff „face“}

Dem Begriff „face“ liegen die menschliche Kommunikation und Interaktion sowie die Beziehungen zwischen den Kommunikationspartnern zugrunde. Diese Beziehungen können wie folgt definiert werden: „Beziehungen sind vielschichtige, unterschiedlich stabile, unterschiedlich dauerhafte und unterschiedlich dynamische Elemente in der Kommunikation. Beziehungen sind zwar in vielen Fällen durch bestimmte Gegebenheiten schon vorstrukturiert, sie sind aber immer neu herzustellen und zu situieren" (Holly 2001: 1384). Den Einfluss auf die Gestaltung der Beziehungen haben Geschlecht, Status sowie persönliche Verhaltensweise und Charakter, weil sie „Ausgangspunkte und Gegenstand von Selbst- und Fremddarstellungen werden können“ (ebd.). In der Kommunikation haben Gesprächspartner die Möglichkeit, eins oder mehrere Bilder von sich selbst zu kreieren. Die Kommunikationspartner verfolgen das Ziel, sich auf eine bestimmte Art und Weise meist 
in einem guten Licht darzustellen, womit der Begriff ,face“ bzw. ,image“ eng zusammenhängt. Ausgehend von Goffmans (1987) Theorie ergibt sich, dass „die Beteiligten bemüht sind, ein 'image of self' zu gewährleisten, dem gesellschaftlich positive Werte inhärent sind“" (Schwitalla 1996: 282). Die von Goffman erarbeitete und entwickelte face-Theorie wurde in der Sprachwissenschaft um weitere Aspekte ergänzt. In diesem Zusammenhang definieren Brown und Levinson (1987: 61) face als ,the public self-image that every member wants to claim for himself“. Das so verstandene face (Gesicht) besteht aus zwei konnexen Aspekten: aus positivem und negativem face, die wie folgt zu verstehen sind:

negative face: the basic claim to territories, personal preserves, rights to non-distraction - i.e. to freedom of action and freedom from imposition

positive face: the positive consistent self-image or 'personality' (crucially including the desire that this self-image be appreciated and approved of) claimed by interactants. (Brown/Levinson 1987: 61)

Das negative Gesicht bezeichnet „de[n] Wunsch nach Selbstbestimmung des Handelns, de[n] Wunsch, über ein eigenes Territorium im konkreten wie im übertragenen Sinne zu verfügen“" (Schwitalla 1996: 282). Die Theorie von Brown/ Levinson (1987) wurde zum Modell der sprachlichen Höflichkeit entwickelt. In diesem Modell wird davon ausgegangen, dass der Sprecher die möglichen Gesichtsbedrohungen seiner eigenen Äußerungen einschätzen kann und diese erst nach einem Kalkül vollzieht. Dabei müssen drei Dimensionen beachtet werden: Macht, Distanz und sozialer Rang. Der Sprecher zielt mit solchem Verhalten auf eine möglichst große Wahrung seines Gesichts ab (vgl. Schwitalla 1996: 282).

Mit der Unterscheidung des positiven und negativen Gesichts hängt das facesaving-Modell zusammen. Unter diesem werden Handlungen verstanden, durch die Gesichtsbedrohungen vermieden werden können. Das positive Gesicht bezeichnet ,den Wunsch nach Anerkennung und Bestätigung des Selbstbildes“ (Lüger 2014: 42), das negative Gesicht bezieht sich indessen auf „den Wunsch eines Individuums, eine bestimmte Distanz, einen Freiraum, ein eigenes Territorium zu bewahren" (ebd.). Hinsichtlich des von mir untersuchten Texts kommen besonders Bedrohungen des positiven Gesichts zur Geltung. Zu gesichtsbedrohenden Handlungen können hier u.a. Kritik oder Vorwurf gezählt werden (vgl. Lüger 2014: 42).

Die oben genannten Dimensionen sollen unter der Berücksichtigung aller Kommunikationspartner untersucht werden. In meinem Beitrag möchte ich mich jedoch vor allem auf die Image-Arbeit des Interviewten sowie die Strategien seiner Selbstdarstellung und der Gesichtswahrung/Gesichtsschonung konzentrieren. Auf die Strategien des Fragenden, d.h. der „Spiegel“-Redaktion, kann ich leider in diesem Beitrag nur kurz eingehen. Auch wenn sich die Untersuchung lediglich auf eine Partei bzw. ein Individuum bezieht, erweist sich face ,als komplexe Verhaltensanforderung: eine Person muss mit mehreren und möglicherweise widerstreitenden Aspekten ihres Selbstbildes fertig werden und je nach Partnerkonstellation, Interesse, Situation und Stand der Interaktion bestimmte Seiten ihrer 
sozialen Präsenz hervorkehren, diese mit anderen 'versöhnen' und andere verdekken“" (Schwitalla 1996: 283).

Zusammenfassend lässt sich face in Anlehnung an Schwitalla (1996: 283 ff.) folgendermaßen charakterisieren:

- Ein Gesicht ist veränderbar, alle Menschen haben mehr als nur ein Gesicht, das in der zwischenmenschlichen Kommunikation variieren kann.

- Das Gesicht hängt einerseits von dem Individuum selbst und seiner Erscheinungsweise $\mathrm{ab}$, andererseits aber auch von der Reaktion und Interpretation der anderen.

Schwitalla (ebd.) unterstreicht zudem, dass sich hinter dem face-Konzept Prozesse und Verfahren verbergen, die auf soziale Anerkennung oder soziale Missachtung abzielen. Das Konzept face eignet sich für Untersuchungen der Beziehungsaspekte und Selbstdarstellungsverfahren im Gespräch wie auch für die Untersuchung der Dialoge, ihrer Organisation, Phasen und Regeln (wie Sprecherwechsel, Beginnen und Beenden von Gesprächen, Zustimmen, Abstreiten, Selbstund Fremdkorrekturen).

\section{Emotionalität in Gesprächen}

Der zweite besonders wichtige Aspekt ist die Emotionalität in dem untersuchten Interview, die sich vorwiegend auf der sprachlichen Ebene und in den Argumentationssträngen widerspiegelt. Da ein Presseinterview der Untersuchung unterzogen wird, können an dieser Stelle besonders expressive und emotionale nonverbale Mittel wie Mimik und Gestik nicht miteinbezogen werden.

Im untersuchten Interview mit Boris Becker hat die Emotionalität eine besonders wichtige Funktion. Zu dieser kann im Allgemeinen Folgendes festgehalten werden: „Zwischen kommunikativem Verhalten und Emotionen bestehen vielfältige Wirkungszusammenhänge und Wechselbeziehungen. Sie sind beidseitig gerichtet: Kommunikatives Verhalten kann Emotionen beeinflussen, sowohl eigene wie auch die anderer Personen, und umgekehrt können Emotionen das eigene Kommunikationsverhalten wie auch das kommunikative Verhalten anderer affizieren und verändern“" (Fiehler 2008: 758).

Interviews mit Prominenten stellen einen großen Spielraum für die Selbstdarstellung des Interviewten dar. Interviews sind ein Frage-Antwort-Spiel, an dem immer mindestens zwei Personen beteiligt sind. Sie tauschen Informationen, ,indem sie sich mittels sprachlicher Handlungen über ein Thema verständigen, sie [tauschen] zugleich auch immer Bewertungen aus, sie [tauschen] sich aus über ihre Einstellungen zu den infrage stehenden Sachverhalten. In diesem Sinne sind Sachverhalte immer bewertende Sachverhalte“ (Fiehler 2008: 759; Hervorh. im Original - J.D.-A.). Dazu führt Fiehler (ebd.) einige Bereiche auf, in denen die 
Manifestation der Emotionen im Gespräch zur Geltung kommt. Dazu werden gezählt:

- physiologische Manifestation (z.B. Zittern),

- nonverbale Manifestation (z.B. Körperhaltung, Lachen),

- verbalisierungsbegleitende Manifestation (z.B. Sprechtempo),

- Manifestation im verbalen Bereich, darunter Wortwahl, Ausrufe, Vorwürfe,

- Manifestation im Gesprächsverlauf (z.B. Wahl des Themas, Verweigerung, Gesprächsmodalität).

Im Rahmen meines Beitrags möchte ich mich auf die Manifestation der Emotionalität im verbalen Bereich konzentrieren. Dabei geht es vor allem um stark emotional-verbale oder verbal-emotionale sprachliche Mittel. Emotional-verbale Mittel sind z.B. kommunikativ relevant verwendete Interjektionen, Ausrufe wie Oh Gott! oder formelhafte Bewertungen meist in Gestalt pragmatischer Phraseologismen wie Ist ja irre! Charakteristisch für sie ist ihre kommunikative und situative Einbettung. Sie sind konventionalisierte Mittel, die erst in der gegebenen Situation ihre Bedeutung entfalten. Die zweite Gruppe, d.h. verbal-emotionale Ausdrücke zeichnen sich dadurch aus, dass bei ihnen die verbale also inhaltlichthematische Komponente dominiert. Diese Mittel eignen sich ausgezeichnet dazu, Vorwürfe, Drohungen oder Klagen zu signalisieren (z.B. Lass das doch jetzt endlich mal!). Allerdings ist darauf hinzuweisen, dass die Grenze zwischen beiden Klassen fließend ist. Der gemeinsame Nenner bleiben Emotion und Emotionalität (vgl. Fiehler 2008: 768).

\section{Analyse}

Ziel meiner Analyse ist es, die oben dargestellten Aspekte face, Image-Arbeit und Emotionalität in einem Interview näher zu erläutern. Wie bereits vorausgeschickt, erweist sich face als ein komplexes Verfahren. Wie sich zeigen wird, können sogar in einem Interview verschiedene Bilder einer Person zur Geltung kommen. Weiterhin kann zugleich der Übergang zwischen konkreten images gezeigt werden. In meiner Recherche möchte ich mich vor allem auf die Selbstdarstellung des Interviewten konzentrieren. Dabei verstehe ich diese Selbstdarstellung nach Schwitalla (1996: 289) als „ein Konzept, das auf bewusste oder unbewusste Effekte bei Rezipienten angelegt ist. Beteiligte entwerfen Selbstbilder in Erwartungen möglicher eigener Wirkungsmöglichkeiten“. Unter dem Begriff „Selbstdarstellung“ wird also das Selbstbildnis verstanden, d.h. die Darstellung der eigenen Person, um seine Fähigkeiten zu zeigen (vgl. Duden Universalwörterbuch).

Gespräche in Publikationsmedien werden durch Mehrfachadressiertheit charakterisiert. Der Interviewte ist sich bewusst, dass seine Aussagen, Meinungen und Darstellungen von der Öffentlichkeit bewertet werden, und eben dadurch „,kommt 
als mögliches Handlungsziel seines sprachlichen Verhaltens die öffentliche Selbstdarstellung ins Spiel“" (Schwitalla 1979: 183; Hervorh. im Original - J.D.-A.). Diese Möglichkeit nutzen vor allem Politiker und Prominente, die ein Interview dazu verwenden, ,über die von ihnen verlangten Informationen, Wertungen und Begründungen hinaus sich selbst, ihre Ziele und Leistungen beim Publikum gut zu 'verkaufen'“ (ebd.). Vor allem Prominente nutzen Interviews dazu, sich in ein gutes Licht zu rücken. Dabei ist noch der Aspekt der Freude und des Vergnügens zu berücksichtigen, die das Publikum bzw. die Öffentlichkeit daran hat, etwas aus dem Privatleben Prominenter zu erfahren und somit an deren Leben quasi teilzunehmen (vgl. Schwitalla 1979: 184). Die oben aufgeführten Aussagen Schwitallas betreffen mündliche, d.h. nicht verschriftlichte Gespräche in Pressemedien. Mir scheint jedoch, dass sie auch auf verschriftlichte Interviews in der Presse bezogen werden können.

Das der Analyse unterzogene Interview kann in Anlehnung an Schwitalla (1979: 329) folgendermaßen charakterisiert werden:

- Ein Interview mit Prominenten ist legerer und freundlicher als ein Interview mit Politikern.

- Im Laufe des Interviews geht es vielmehr um ein Gespräch über das Leben, die Karriere des Interviewten als um Sachverhalte schlechthin.

- Der Interviewte wird nicht nur an objektiven Errungenschaften und Leistungen gemessen (hier als Tennisspieler), sondern auch daran, was ihn privat anbelangt (in unserem Fall finanzielle Schwierigkeiten, Alkoholprobleme, Lebensstil etc.).

Ganz am Anfang des untersuchten Interviews mit dem Titel „Ich bin der Drachentöter“ wird vom „Spiegel“" angekündigt, dass das Gespräch dem Erfolg Beckers gewidmet wird. Diese Feststellung erweckt beim Leser den Eindruck, dass das nun vorliegende Interview tatsächlich das erfolgreiche Leben Beckers betreffen wird. Wie die Lektüre zeigt, konzentriert sich das Interview jedoch auf Skandale, finanzielle Probleme und Misserfolge des Interviewten. Das Gespräch enthält vor allem Unterstellungen, heikle Behauptungen und Fragen, die Becker nicht gelegen kommen und gegen die er sich wehrt. In den untersuchten Textpassagen zeigen sich viele Stellen, an denen Becker sich wehrt, die Fragen nicht beantwortet, die Schuld anderen zuschiebt, den Spieß umdreht oder Tatsachen leugnet.

Welches Selbstbild möchte Becker in dem herangezogenen Interview entwerfen? Welche Strategien verfolgt er, um sich in ein gutes Licht zu rücken, seinen Ruf zu reparieren und zu verteidigen? Und welche sprachliche Mittel verwendet er, um sein Gesicht zu wahren? Diesen Fragen möchte ich im Folgenden nachgehen. Dazu werden Textpassagen angeführt. Textstellen, die bedeutsam zur Herausarbeitung einer bestimmten Selbstdarstellung führen, werden von mir fettgesetzt. Alle Textabschnitte werden anschließend besprochen. 
Im Interview „Ich bin der Drachentöter“ kreiert Boris Becker folgende Selbstbilder:

- Opfer / Benachteiligter

SPIEGEL: Herr Becker, wir wollen mit Ihnen über Erfolg reden.

Becker: Klingt doch gut.

SPIEGEL: Es ist 29 Jahre her, dass Sie Wimbledon gewonnen haben...

Becker: ...zum einen ein Moment, den ich mir immer erträumt habe, zum anderen einer, an dem ich ein Monster erschuf: den 17-jährigen Leimener. Es ist wie bei der ersten Mondlandung, da erinnern sich die Leute auch noch, wo sie damals waren. Ich habe mich immer gewehrt zu sagen: „Ich bin ein Held“ oder „Ich bin eine Legende“. Ich bin nur ein Mensch. Mit Fehlern. Ich mache jeden Tag fünf Stück. Nur werde ich dafür immer öffentlich gekocht.

SPIEGEL: Irgendeine Idee, wieso das so ist?

Becker: Vielleicht enttäuschte Liebe. Viele Deutsche, vor allem Männer, haben mich wie einen Sohn gesehen. Das ist ein völlig falscher Ansatz. Ich hatte einen tollen Vater, ich wollte kein Sohn von anderen Vätern sein. Diese Menschen haben eine genaue Vorstellung davon, was ich sein soll: ein Tennisdenkmal oder ein Boulevardpirat. Die wollen den 46-jährigen Unternehmer Boris Becker gar nicht kennenlernen.

SPIEGEL: Meinen Sie wirklich? Ist es nicht eher so, dass die Leute, die früher mit Ihnen mitfieberten, heute Mitleid haben?

Becker: Warum sollten sie? Es geht mir gut.

SPIEGEL: Weil Sie im Fernsehen dämliche Hüte aufsetzen und sich zum Affen machen. Die Leute fragen sich: Warum, um Himmels willen, benimmt sich Boris Becker nicht wie ein erwachsener Mann?

Becker: Ja, da schenke ich mir erst einmal etwas Wasser ein, bevor ich zur Antwort aushole. Also, ich lebe mein Leben nicht, um es anderen recht zu machen. Ich versuche, niemandem zu schaden. Ich versuche, meinen Mitmenschen mit Respekt und mit Anerkennung zu begegnen. Ich nehme den Leuten nichts weg. Ich habe niemanden gezwungen, die Sendung einzuschalten, die ich übrigens amüsant fand. Offenbar hat nicht jeder meinen Humor.

In der oben angeführten Textpassage vergleicht Becker seinen WimbledonSieg mit der ersten Mondlandung und verleiht somit seiner Errungenschaft eine historische Bedeutung. Er stellt sich in seiner Aussage als ein Mensch wie du und ich dar, der Fehler begeht und anderen Menschen nahesteht. Becker sagt Ich werde [...] öffentlich gekocht; Die wollen den 46-jährigen Unternehmer Boris Becker gar nicht kennenlernen. Er stellt sich als Opfer des öffentlichen Interesses dar, indem er konstatiert, dass er selbst niemandem schadet, den anderen nichts wegnimmt und seine Mitmenschen respektiert. Der Interviewte präsentiert sich als bescheidene Person, die nicht als Held oder Legende angesehen werden will. Andererseits wirft der ,Spiegel“-Redakteur Becker vor, er verhalte sich dämlich und mache sich zum Affen, weswegen Menschen mit ihm Mitleid haben. Dem widerspricht Becker eindeutig und sagt Warum sollten sie? Es geht mir gut!

Die zweite Textpassage betrifft von Becker verfasste und vielleicht nicht immer gut durchdachte Tweets. In ihr zeigt sich Becker ebenfalls als Opfer / Benachteiligter: 
SPIEGEL: Heute schon getwittert?

Becker: Nein, wieso? Wo ist das Problem? Ja, ich habe relativ früh erkannt, dass die sozialen Netzwerke eine unglaubliche Kraft haben. Ich will meine Meinung äußern, schreibe einen kurzen Text und erreiche damit sehr viele Leute. Ist doch genial.

SPIEGEL: An Oliver Pocher haben Sie geschrieben: „... wünsche Dir endlich mal ein ECHTES Weib... bist wohl noch etwas jung." Können Sie verstehen, dass man so etwas peinlich findet?

Becker: Es ist doch mein Bier, wenn ich mich bei Twitter mit Oliver Pocher süffisant austausche. Ich bin kein Politiker, mit meinen Tweets möchte ich unterhalten, da gehört es dazu, dass ich auch mal aus der Hüfte schieße. Lasst doch die Kirche im Dorf, Freunde! Komischerweise sind diejenigen, die mich ständig dafür kritisieren, die Schlimmsten. Die schließen von ihrem traurigen Leben auf das anderer. Die sollen mich doch in Ruhe lassen.

Auf die Frage, ob Becker heute schon getwittert hat, erwidert der Interviewte emotional mit einem Vorwurf Nein, wieso? Wo ist das Problem?, als ob er sich wehren möchte. Die Feststellung, seine Veröffentlichungen auf Twitter seien peinlich, hat zur Folge, dass sich Becker besonders erregt zeigt. Am Ende dieser Gesprächssequenz dreht Becker den Spieß um und sagt Komischerweise sind diejenigen, die mich ständig dafür kritisieren, die Schlimmsten.

Auf sprachlicher Ebene scheint diese Passage besonders interessant, weil sie reich an vorwiegend umgangssprachlichen und sehr emotionalen Phraseologismen ist. Diese sind:

- Es ist doch mein Bier!

- Da gehört es dazu, dass ich auch mal aus der Hüfte schieße.

- Lasst doch die Kirche im Dorf, Freunde!

- Die sollen mich doch in Ruhe lassen.

In diesem Zusammenhang ist festzustellen, dass den hier präsenten Phraseologismen eine wichtige Rolle zukommt, indem sie ,der Themen- und Handlungsstrukturierung, der Sachverhaltsdarstellung und der Beziehungsgestaltung dienen können. Weitere Möglichkeiten sind die Funktionen der Selbstdarstellung sowie der Adressatenberücksichtigung“" (Skod-Södersved 2007: 270).

Vor dem Hintergrund der Gerüchte über finanzielle Probleme Beckers taucht die Frage nach seiner Tätigkeit als Tennistrainer auf, was im folgenden Textabschnitt thematisiert wird. In dieser Textpassage zeigt sich Becker als:

- Guter Coach

SPIEGEL: Warum haben Sie das Angebot angenommen, für ihn als Trainer zu arbeiten?

Becker: Glauben Sie mir, es war in meiner Lebensplanung nicht wirklich vorgesehen, mit Novak um die Welt zu reisen. Es gefällt auch meinen Geschäftspartnern nicht, dass ich wochenlang unterwegs bin. Aber die erste Liebe rostet nicht. Tennis ist meine große Leidenschaft, und wenn ich eines kann, dann Tennis. Kommentieren, analysieren, es den jungen Spielern beibringen. Das ist wie Zähne putzen.

SPIEGEL: Am Geld lag's nicht?

Becker: Es ist nicht der Grund, warum ich das mache. Novak war ja nicht der erste Spieler, der mich angerufen hat. Es gab andere Anfragen, auch von bekannten Spielern.

SPIEGEL: Von wem denn? 
Becker: Verrate ich nicht. Ich war höflich und habe vorgegeben, ich hätte keine Zeit. Ehrlich gesagt, war der wahre Grund ein anderer: Es hätte sich nicht gelohnt. Die Jungs hatten nicht die Qualität, sie dahin zu bringen, wo ich sie gern gehabt hätte. Als Novak anrief, wusste ich: Der hat's drauf! Wenn der ein paar Kleinigkeiten ändert, kommt er wieder nach ganz oben.

Becker bestreitet, der Grund seiner Tätigkeit als Coach sei das Geld. Er vergleicht die Beschäftigung mit Tennis mit der ersten Liebe, mit etwas, was ihm natürlich und offensichtlich scheint. Um seine Argumentation zu stützen, führt Becker an, dass er schon früher Anfragen von anderen Tennisspielern bekommen habe. Die sollten jedoch für ihn nicht genügend Qualifikationen gehabt haben. Auf die Nachfrage des Interviewers, um welche Spieler es sich handelt, erwidert Becker nachdrücklich Verrate ich nicht. Der Leser kann somit nur spekulieren, ob es tatsächlich irgendwelche Anfragen gab.

Den Wendepunkt im Interview bringt die unten angeführte Passage, in der sich Becker zu einem Fehler bekennt:

- Bekenner

SPIEGEL: Das Angebot von Djoković kam zu einem Zeitpunkt, als gerade eine Welle der Kritik über Ihnen zusammenbrach, ausgelöst durch Ihre Autobiografie. Von einer ,deutschen Tragödie“" war die Rede, von einer „Selbstdemontage“. Sie waren nicht länger „Bumm-BummBoris“, sondern „Dumm-Dumm-Boris“. Man könnte meinen, Sie profitieren mehr von der Zusammenarbeit als Djoković.

Becker: Ach was! Ich gebe zu, ich habe andere Reaktionen auf das Buch erwartet. Im Nachhinein muss ich sagen: Das Buch war ein Fehler. Mein Leben als Geschäftsmann, der glücklich verheiratet ist, findet die Öffentlichkeit offensichtlich nicht spannend. Die liest lieber andere Überschriften. Nur weil ich in die Pocher-Show gegangen bin, weil es ein unvorteilhaftes Foto von mir gab, bin ich jetzt ein schlechter Mensch, habe einen miesen Charakter, bin arbeitslos, vernachlässige meine Kinder und bin auf der Suche nach Anerkennung.

Das ist doch Wahnsinn.

SPIEGEL: Das wirkliche Leben ist komplizierter als das Tennisleben?

Becker: Schon als Tennisspieler habe ich provoziert, aber ich konnte mich mit dem Argument rechtfertigen: Einen Wimbledon-Sieger kritisiert man nicht, ich bin Siegfried, der Drachentöter, ich kann alles, ihr könnt mich mal. Dieses Argument ist mir entfallen. Es ist grundsätzlich schwierig für Sportler, die nach ihrer Karriere nicht unbedingt morgen wieder arbeiten müssen, etwas zu finden, das sie wirklich ausfüllt. Wo man sich zu Hause fühlt. Das war auch bei mir der Fall.

Bis auf diese Stelle im Interview bestreitet Becker alle Argumente und weist Vorwürfe zurück. Nur einmal bekennt er einen Fehler: Ach was! Ich gebe zu, ich habe andere Reaktionen auf das Buch erwartet. Im Nachhinein muss ich sagen: Das Buch war ein Fehler. Direkt danach zeigt sich Becker erneut als Opfer und macht wieder andere Menschen für diesen Misserfolg verantwortlich, weil die Öffentlichkeit am liebsten Skandalgeschichten über ihn liest und nicht seine Bücher. Sein Statement konstatiert er mit Das ist doch Wahnsinn. In dieser Passage möchte Becker sein menschliches Gesicht zeigen, er sei nicht mehr der früher erwähnte Drachentöter und zeigt sich als Sportler, dem es nach der Beendigung der Karriere schwerfällt, sich im normalen Leben zurechtzufinden. 
Im weiteren Verlauf des Interviews präsentiert sich Becker als:

- Abstreiter

SPIEGEL: Wo läuft es denn gerade ökonomisch besonders gut? Bei Ihren drei Autohäusern in Mecklenburg-Vorpommern, die Sie immer als Basis Ihrer Geschäfte bezeichnet haben, ja wohl nicht.

Becker: Nein, das ist faktisch falsch.

SPIEGEL: In der Bilanz für 2012 steht ein Minus von 208 083,84 Euro.

Becker: Wenn ich Ihnen die Zahlen heute nenne, würden Sie überrascht sein. Was Sie da haben, ist ein Zwischenbericht.

SPIEGEL: Herr Becker, die Bilanz wurde im Bundesanzeiger veröffentlicht.

Becker: Okay, gut. Was wollen Sie?

SPIEGEL: Wissen, wo es ökonomisch gut läuft. Mit den Autohäusern läuft es vielleicht nicht mehr so gut.

Becker: Also, ich sehe das anders. Ich habe die Zahlen vor zwei Wochen gesehen, und die sind absolut wunderbar. Für mich stellt sich die Frage: Bin ich im Herzen ein Autoverkäufer? Verkaufe ich die Häuser lieber? Oder fusioniere ich mit anderen Häusern, damit ich größer und rentabler werde? Da bin ich mit Mercedes-Benz im Gespräch. Im Herbst treffen wir eine Entscheidung.

SPIEGEL: Anders gefragt: Es hieß in letzter Zeit öfter, Sie seien so gut wie pleite. Sind Sie pleite? Wovon leben Sie?

Becker: Macht euch keine Sorgen um mich. Nicht jeder Deal hat funktioniert, aber 51 Prozent. Ich finde es eine Unverschämtheit, dass sich Menschen darüber Gedanken machen. Dass Sie das wissen wollen, ist okay, das ist Ihr Job. Aber ich stehe hier ja nicht vor Gericht und muss Ihnen sagen, was ich im Monat verdiene. Wissen Sie was? Ich bin niemandem Rechenschaft schuldig. Es geht niemanden etwas an. Punkt! Ich lebe in London, und hier habe ich drei Joint Ventures. Es sind summa summarum 50 Menschen für und mit Boris Becker im Einsatz.

SPIEGEL: Übertreiben Sie nicht ein bisschen?

Becker: Offen gestanden, nervt es mich schon, dass mir das in Deutschland kaum einer glaubt.

Viel Aufmerksamkeit wird im Interview der finanziellen Lage und den misslungenen Geschäften Beckers gewidmet. Es wird zwar anfangs angekündigt, das Gespräch handelt von Beckers Erfolg, den größten Teil davon machen jedoch Erwägungen über Misserfolge und Finanzen aus. In dieser Passage ist interessant, wie Becker mit Fakten umgeht. Auf die Frage nach der finanziellen Lage und Misserfolge im Autohandel erwidert Becker nachdrücklich Nein, das ist faktisch falsch. Dann führt „Spiegel“ einige Zahlen an, die von Becker wieder in Frage gestellt werden, indem er feststellt Wenn ich Ihnen die Zahlen heute nenne, würden Sie überrascht sein. Der Redakteur zeigt sich aber hartnäckig und hakt weiter nach. Erst nach dem Argument, dass seine Bilanz im Bundesanzeiger veröffentlicht wurde, gibt Becker dem „Der Spiegel“ recht (Okay, gut. Was wollen Sie?). In diesem Abschnitt kommt zur Geltung, dass sich Becker nicht eindeutig dazu äußern will, ob er pleite ist. Er umgeht die Frage und sagt Macht euch keine Sorgen um mich und geht mit Wissen Sie was? Ich bin niemandem Rechenschaft schuldig. Es geht niemanden etwas an. Punkt! zum Angriff über. Becker verweist darauf, dass andere es wieder auf ihn abgesehen haben: Offen gestanden, nervt es mich schon, dass mir das in Deutschland kaum einer glaubt. 
Im Interview zeigt sich Becker sehr gern als erfolgreicher Geschäftsmann:

- (geheimnisvoller) Geschäftsmann und Spezialist SPIEGEL: Mit welchen Unternehmen kooperieren Sie in London?

Becker: Ich mache Geschäfte mit einer Investmentbank. Den Namen möchte ich aus Diskretion hier nicht nennen. Wir haben gemeinsam Büros fast auf der ganzen Welt. Ich vermittle Geschäfte im Bereich Immobilien, Technologie, Bodenschätze und Neue Medien. Sozusagen Mergers and Acquisitions: Ich bringe A und B zusammen. Dafür stehe ich jeden Morgen auf, ohne da jetzt im Detail mehr drauf einzugehen.

SPIEGEL. Schade. Um wen handelt es sich bei den anderen Firmen?

Becker: Ich arbeite mit der Sportmarketing-Agentur Doyen Global zusammen. [...] Die Firma vertritt bekannte Leute aus Sport, Politik und Kultur. Die kümmern sich global um die Marke Boris Becker.

SPIEGEL: Und wie positioniert man die?

Becker: Meinen Namen bringt man mit Sport, Internationalität, Erfolg, Dauerhaftigkeit und, ob Sie es glauben oder nicht, mit einem modernen Deutschland in Verbindung. Ich halte Vorträge bei Kongressen und Konferenzen.

SPIEGEL: $\mathrm{Zu}$ welchem Thema sprechen Sie?

Becker: Ich referiere über die Bedeutung von Sport. Über Gesundheit, Motivation, Ernährung. Wie wird man ein Gewinner? Da hilft ein Wimbledon-Sieg als Trainer natürlich enorm. Aber wissen Sie was?

SPIEGEL: Was?

Becker: Ich halte 20 Vorträge im Jahr, überall werde ich gebucht, nur nicht in Deutschland. Wenn ich Ihnen Beispiele nenne, werden Sie denken: Das kann nicht sein!

SPIEGEL: Lassen Sie hören.

Becker: Ich war 2013 bei Doha Goals in Katar. Das ist das Weltwirtschaftsforum für Sport, ich war einer der Hauptredner. Auch in Afrika werde ich gebucht. Ich habe vor jungen Führungskräften in Johannesburg geredet und war zuletzt in Gabun auf einem Businesskongress. Zwei Tage nach den U.S. Open trete ich in Hongkong auf, aber weder in Hamburg noch in München, noch in Osnabrück. Es ist irre.

Fragwürdig und nicht überzeugend wirken einige Stellen im Gespräch, in denen der Interviewte zwar einige Informationen preisgibt, jedoch geheimnisvoll bleibt. Er lässt den Leser vermuten, wie seine tatsächliche Lage ist. Becker soll einige Geschäfte in London führen, welche genau es sind, möchte er nicht verraten. Er sagt ohne da jetzt im Detail mehr drauf einzugehen und schickt ein klares Signal, dass er zu diesem Bereich nicht mehr befragt werden möchte. Er stellt sich wieder als Person dar, die mit Erfolg assoziiert wird: Meinen Namen bringt man mit Sport, Internationalität, Erfolg, Dauerhaftigkeit [...] in Verbindung. Des Weiteren verweist er darauf, dass sein Erfolg in Deutschland nicht richtig eingeschätzt wird.

Darüber hinaus möchte Becker nicht mehr als jemand, der nur für Skandale sorgt, angesehen werden:

- anständige Person

SPIEGEL: Ihre Finca auf Mallorca wäre im Juni fast zwangsversteigert worden. Wäre es ein Drama, wenn Sie sagen würden, ich bin erfolgreich und zufrieden, aber hier und da geht auch richtig was schief?

Becker: Wenn es stimmen würde, wäre es kein Problem, aber es stimmt nicht. Es ist eine private Immobilie, wie ich noch einige besitze. Und was ich mit dieser Finca mache, ob ich sie 
verschenke, verkaufe, meinen Kindern überlasse: Das ist doch meine freie Entscheidung. Ich habe meinen Umsatz, meinen Profit - Ende Gelände!

SPIEGEL: Klar, Sie könnten die Finca auch niederbrennen, wenn es Ihnen Spaß macht. Aber es geht doch um Ihren Ruf. Sie sollen jahrelang den Gärtner, den Hausmeister und Bauarbeiter nicht bezahlt haben.

Becker: Wer sagt denn, dass das stimmt?

SPIEGEL: Stimmt es also nicht?

Becker: Nein. Diese Skandalgeschichten über mich werden immer langweiliger, weil es olle Kamellen sind. Da meldet sich ein Hausmeister, es ist Sommerloch, dann kommt der eben in die Zeitung. Was ja ein Unding ist. Wir haben Kriege in der Welt, echte Probleme, und so einer kriegt Platz. Einen Teufel werde ich tun, das zu kommentieren. Da kassiere ich nur wieder einen Return. Das habe ich mittlerweile gelernt: die Klappe zu halten.

Während der Lektüre des Interviews gewinnt man den Eindruck, der Redakteur zielt darauf ab, dass Boris Becker endlich zugibt, in einigen Sphären seines Lebens doch nicht so erfolgreich zu sein, wie er von sich behauptet. Dem widerspricht Becker mit der Erklärung, dass das nicht stimmt, und schließt seine Aussage mit der expressiven und emotionalen Formel Ende Gelände! Im weiteren Verlauf des Interviews wird Becker nach einer Skandalgeschichte gefragt, laut der er seinen Arbeiter auf Mallorca nicht bezahlt habe. Das bestreitet er damit, dass es sich um olle Kamellen handelt und er schon gelernt hat, in solchen Fragen die Klappe zu halten, was der Aussage einen umgangssprachlichen Charakter verleiht. In der unten aufgeführten Textpassage versucht Becker seinen aufgrund der Skandale verlorenen Ruf zurückzugewinnen:

- Workaholic

SPIEGEL: Seit Januar sind Sie kein Markenbotschaftermehr für Mercedes, der Autohersteller war Ihr treuester Partner. Auch der Werbevertrag mit PokerStars ist ausgelaufen. Als Testimonial sind Sie nicht mehr gefragt.

Becker: Es war nie mein Berufswunsch, Werbeträger zu sein.

SPIEGEL: Das ist aber lukrativ.

$[\ldots]$

Becker: [...] Ich habe gern mit Mercedes-Benz gearbeitet, mein erstes Auto war ein Mercedes 190 E, und ich bin auch abergläubisch: Mir ist in einem Mercedes noch nie etwas passiert, als Fahrer oder Beifahrer. Und ich fahre weiß Gott sehr schnell.

SPIEGEL: Deswegen auch die vielen Strafzettel, die Sie angeblich nicht bezahlt haben.

Becker: Wenn ich die nicht bezahlt hätte, hätte das doch sicherlich erhebliche Konsequenzen für mich gehabt. Man muss solche Geschichten auch mal zu Ende denken. Wenn das alles stimmen würde, was über mich erzählt und geschrieben wird, wie könnte ich denn dann so leben, wie ich es jetzt tue?

SPIEGEL: Den halben Tag Golf spielen.

Becker: Tue ich ja nicht.

SPIEGEL: Könnten Sie es?

Becker: Wenn ich meine Anteile und Besitztümer verkaufen würde, dann könnte ich das. Will ich aber nicht. Ich arbeite gern. Ich bin fast ein Workaholic. So funktioniere ich, so habe ich auch Tennis gespielt. Ich habe mich auch mal treiben lassen - Mädels, Golfspielen und fast jede Nacht auf einer Party. Aber das ist lange her. Für mich war das unbefriedigend, was nicht am Mädel lag. Das ist nicht mein Ding. 
Becker verlor lukrative Werbeverträge, obgleich Werbeträger zu sein angeblich nie sein Wunsch war. Um seinen Ruf zu verteidigen, stellt sich Becker als Workaholic dar. Er möchte sein Image wahren, indem er sagt, er arbeite gern. Früher war er als Spaßvogel bekannt - Mädels, Golfspielen und fast jede Nacht auf einer Party. Becker will nicht länger diesen Ruf haben und mit einem solchen Leben abgeschlossen haben, indem er erklärt Das ist nicht mein Ding.

Das Interview schließt mit Fragen nach Beckers Verhältnis zu Deutschland:

- Patriot

SPIEGEL: Hinter vorgehaltener Hand heißt es: Der Becker ist dick geworden, der hat auch ein aufgeschwemmtes, rotes Gesicht, der säuft bestimmt.

Becker: Ja. Ja, klar. Diese Schadenfreude! Was ist denn das? Was ist das für eine Mentalität, sich über Kranke und Verletzte das Maul zu zerreißen? Mit solchen Leuten muss ich nicht reden. Da muss ich auch nichts klarstellen.

SPIEGEL: Mögen Sie Deutschland?

Becker: Ich bin nach wie vor Patriot, ich bekomme Gänsehaut, wenn ich die Nationalhymne höre, und ich habe mich gefreut, als Deutschland Weltmeister geworden ist. Trotzdem ist ein gewisser Abstand eingetreten. Das Problem ist, in Deutschland ruft Erfolg sofort Neid hervor. Überall sonst ist das anders.

SPIEGEL: Ein deutsches Idol zu sein ist die Pest, so hören Sie sich an.

Becker: Das ist mir zu hart ausgedrückt. Und es ist auch falsch. Aber wie viele Idole haben wir denn? Und wo leben die? Franz Beckenbauer in Österreich. Heidi Klum und Steffi Graf in den USA. Sebastian Vettel in der Schweiz, Nico Rosberg in Monaco. Was ist wohl der Grund dafür?

SPIEGEL: Vielleicht sind die Steuern dort einfach niedriger?

Becker: Denken Sie, dass ich in London keine Steuern zahlen muss? Ich lebe doch nicht auf den Cayman Islands. Es ist keine Steuerflucht, es ist eine Flucht in Frieden und Freiheit. So weit, so gut?

SPIEGEL: So weit, so gut. Herr Becker, wir danken Ihnen für dieses Gespräch.

Im ganzen Interview widerspricht Becker allen Unterstellungen, es sei pleite und weist Skandalgeschichten und Gerüchte zurück. Er behauptet, Deutsche haben es auf ihn abgesehen, seien neidisch auf seinen Erfolg und interessieren sich nur für seine Misserfolge und Niederlagen. Dies bekräftigt er auch in dem angeführten Abschnitt, indem er konstatiert Ja. Ja, klar. Diese Schadenfreude! Was ist denn das? Er erklärt sich zum Patrioten, allerdings mit einer Einschränkung (Trotzdem ist ein gewisser Abstand eingetreten. Das Problem ist, in Deutschland ruft Erfolg sofort Neid hervor). Auf den Vorwurf, er lebe in London, weil die Steuern dort niedriger sind, reagiert Becker empört: Denken Sie, dass ich in London keine Steuern zahlen muss? Ich lebe doch nicht auf den Cayman Islands. Es ist keine Steuerflucht, es ist eine Flucht in Frieden und Freiheit. So weit, so gut? und schließt somit selbst das Interview ab, was eher unüblich ist, denn Interviews werden meist vom Interviewer beendet. Mit der Formel So weit, so gut? lenkt Becker das Gespräch in die von ihm gewünschte Bahn und gibt zugleich das klare Signal, an dieser Stelle das Interview abschließen zu wollen. 


\section{Auswertung}

Eine genauere Lektüre der Interviewpassagen zeigt, dass sich Becker sehr verschieden profiliert und unterschiedliche Selbstbilder kreiert. Er zeigt sich als hartnäckiger Sprecher, der seine Fehler nicht eingesteht. Nur einmal legt er ein Eingeständnis ab, indem er feststellt, dass seine Autobiografie tatsächlich ein Fehler war. Die Selbstdarstellung Beckers entfaltet sich im Laufe des Interviews. In linguistischer Sicht konnten im untersuchten Interview sprachliche Mittel und Strukturen ermittelt werden, die der Wahrung der oben genannten Selbstbilder dienen. Becker stellt sich in dem Interview als Spezialist, guter Trainer und Geschäftsmann dar, der angeblich trotz schwieriger Umstände doch nicht in finanziellen Schwierigkeiten ist, und als Person, die von anderen Menschen durch ständiges Kritisieren oder unwahre und präparierte Skandalgeschichten eigentlich benachteiligt wird. Diese Strategie wird mit dem Ziel vorgenommen, sich als erfolgreicher Mensch und Legende darzustellen. Die ermittelten Selbstdarstellungen sollen das positive Gesicht Beckers, also den Wunsch nach Anerkennung, wahren und bestätigen.

In dem theoretischen Teil meines Beitrags habe ich darauf hingewiesen, dass sich faces aus der Kommunikation ergeben. Das ist von einer hervorragenden Bedeutung besonders für Interviews, an denen sich immer mindestens zwei Personen beteiligen. Das gewonnene face des Interviewten ergibt sich aus dem Zusammenspiel zwischen Fragen und auf sie erteilten Antworten. Interessanterweise ergeben sich dem positiven wie auch dem negativen Gesicht dienende Selbstdarstellungen Beckers aus den Fragen, in denen von der Redaktion des „Spiegels“ etwas völlig anderes behauptet wird als das, was Becker von sich preisgibt. So wird in den Fragen oder Feststellungen der Redaktion explizit oder implizit behauptet, Becker sei alkoholsüchtig, pleite, bezahle nicht seine Mitarbeiter, löse Skandale aus. Dem setzt der Interviewte sein Selbstbildnis entgegen. Von sich behauptet Becker, er sei anständiger Mensch, guter Vater, Patriot, ihm ginge es finanziell gut, auch wenn seiner Bilanz etwas anderes zu entnehmen ist. Dieses Bild ergibt sich aus den Strategien, die er im Laufe des Interviews verwendet. Handlungen, die in diesem Fall der Selbstdarstellung dienen, sind u.a.

- abstreiten,

- angreifen,

- vorwerfen,

- sich verteidigen,

- zustimmen,

- Fragen umgehen,

- zurückweisen,

- Antwort verweigern.

Diese Zusammenstellung macht ersichtlich, dass das Gesicht in und durch die Interaktion definiert wird. Der Interviewte und der Interviewende wahren ihre Ge- 
sichter, führen aber gleichzeitig Handlungen aus, die das Gesicht des Gesprächspartners bedrohen sollen. Einige Beispiele werden unten tabellarisch aufgeführt:

\begin{tabular}{|c|c|}
\hline $\begin{array}{c}\text { Darstellung des ,Spiegels“" } \\
\text { (Frage/Behauptung) }\end{array}$ & $\begin{array}{l}\text { Standpunkt Beckers } \\
\text { (Antwort) }\end{array}$ \\
\hline $\begin{array}{l}\text { Meinen Sie wirklich? Ist es nicht eher so, dass } \\
\text { die Leute, die früher mit Ihnen mitfieberten, } \\
\text { heute Mitleid haben? }\end{array}$ & $\begin{array}{l}\text { Warum sollten sie? Es geht mir gut. } \\
\text { (Abstreiten) }\end{array}$ \\
\hline $\begin{array}{l}\text { An Oliver Pocher haben Sie geschrieben: „[...] } \\
\text { wünsche Dir endlich mal ein ECHTES Weib } \\
{[\ldots] \text { bist wohl noch etwas jung.“ Können Sie }} \\
\text { verstehen, dass man so etwas peinlich findet? }\end{array}$ & $\begin{array}{l}\text { Es ist doch mein Bier, wenn ich mich bei Twit- } \\
\text { ter mit Oliver Pocher süffisant austausche. [...] } \\
\text { Lasst doch die Kirche im Dorf, Freunde! } \\
\text { (Verteidigung, Angreifen) }\end{array}$ \\
\hline $\begin{array}{l}\text { Warum haben Sie das Angebot angenommen, } \\
\text { für ihn als Trainer zu arbeiten? [...] Am Geld } \\
\text { lag's nicht? }\end{array}$ & $\begin{array}{l}\text { Es ist nicht der Grund, warum ich das mache. } \\
\text { Novak war ja nicht der erste Spieler, der mich } \\
\text { angerufen hat. Es gab andere Anfragen, auch } \\
\text { von bekannten Spielern. } \\
\text { (Abstreiten) }\end{array}$ \\
\hline $\begin{array}{l}\text { Das Angebot von Djoković kam zu einem Zeit- } \\
\text { punkt, als gerade eine Welle der Kritik über } \\
\text { Ihnen zusammenbrach, ausgelöst durch Ihre } \\
\text { Autobiografie. Von einer „,deutschen Tragödie“ } \\
\text { war die Rede, von einer „Selbstdemontage“. } \\
\text { Sie waren nicht länger „Bumm-Bumm-Boris“, } \\
\text { sondern „Dumm-Dumm-Boris“. Man könnte } \\
\text { meinen, Sie profitieren mehr von der Zusam- } \\
\text { menarbeit als Djoković. }\end{array}$ & $\begin{array}{l}\text { Ach was! } \\
\text { (Abstreiten, Empörung) }\end{array}$ \\
\hline $\begin{array}{l}\text { Wo läuft es denn gerade ökonomisch beson- } \\
\text { ders gut? Bei Ihren drei Autohäusern in Meck- } \\
\text { lenburg-Vorpommern, die Sie immer als Basis } \\
\text { Ihrer Geschäfte bezeichnet haben, ja wohl } \\
\text { nicht. }\end{array}$ & $\begin{array}{l}\text { Nein, das ist faktisch falsch. } \\
\text { (Abstreiten, Verteidigung) }\end{array}$ \\
\hline $\begin{array}{l}\text { In der Bilanz für } 2012 \text { steht ein Minus von } 208 \\
\text { 083,84 Euro. }\end{array}$ & $\begin{array}{l}\text { Wenn ich Ihnen die Zahlen heute nenne, wür- } \\
\text { den Sie überrascht sein. Was Sie da haben, ist } \\
\text { ein Zwischenbericht. } \\
\text { (Umgehen, Zurückweisen) }\end{array}$ \\
\hline $\begin{array}{l}\text { Herr Becker, die Bilanz wurde im Bundesanzei- } \\
\text { ger veröffentlicht. }\end{array}$ & $\begin{array}{l}\text { Okay, gut. Was wollen Sie? } \\
\text { (erzwungenes Zustimmen) }\end{array}$ \\
\hline $\begin{array}{l}\text { Anders gefragt: Es hieß in letzter Zeit öfter, Sie } \\
\text { seien so gut wie pleite. Sind Sie pleite? Wovon } \\
\text { leben Sie? }\end{array}$ & $\begin{array}{l}\text { Macht euch keine Sorgen um mich. } \\
\text { (Umgehen) }\end{array}$ \\
\hline Übertreiben Sie nicht ein bisschen? & $\begin{array}{l}\text { Offen gestanden, nervt es mich schon, dass mir } \\
\text { das in Deutschland kaum einer glaubt. } \\
\text { (Abstreiten, Vorwerfen) }\end{array}$ \\
\hline
\end{tabular}




\begin{tabular}{|l|l|}
\hline $\begin{array}{l}\text { Wäre es ein Drama, wenn Sie sagen würden, } \\
\text { ich bin erfolgreich und zufrieden, aber hier und } \\
\text { da geht auch richtig was schief? }\end{array}$ & $\begin{array}{l}\text { Wenn es stimmen würde, wäre es kein Problem, } \\
\text { aber es stimmt nicht. } \\
\text { (Abstreiten) }\end{array}$ \\
\hline $\begin{array}{l}\text { Sie sollen jahrelang den Gärtner, den Hausmeis- } \\
\text { ter und Bauarbeiter nicht bezahlt haben. }\end{array}$ & $\begin{array}{l}\text { Wer sagt denn, dass das stimmt? } \\
\text { (Zurückweisen, Umgehen) }\end{array}$ \\
\hline $\begin{array}{l}\text { Hinter vorgehaltener Hand heißt es: Der Beck- } \\
\text { er ist dick geworden, der hat auch ein auf- } \\
\text { geschwemmtes, rotes Gesicht, der säuft bes- } \\
\text { timmt. }\end{array}$ & $\begin{array}{l}\text { Ja. Ja, klar. Diese Schadenfreude! Was ist denn } \\
\text { das? } \\
\text { (Abstreiten, Empörung) }\end{array}$ \\
\hline
\end{tabular}

Der letzte Punkt, den ich in der Auswertung präsentieren möchte, ist die sprachliche Realisierung der genannten Selbstdarstellungen. Folgender Überblick lässt die angenommene emotionale Ausprägung des untersuchten Interviews bestätigen. Es handelt sich dabei um emotionale Formeln und Ausdrücke (darunter Phraseologismen und umgangssprachlicher Wortschatz): Wo ist das Problem? / Es ist doch mein Bier. / Lasst doch die Kirche im Dorf, Freunde! / Ach was! / Das ist doch Wahnsinn. / Punkt! / Das kann nicht sein! / Ende Gelände! / Ja. Ja, klar. Diese Schadenfreude! / Was ist denn das? / Ist doch genial! / Es ist irre! / Weiß Gott / um Himmels willen / einen Teufel tun / sich zum Affen machen / dämliche Hüte aufsetzten / aus der Hüfte schießen / in Ruhe lassen / sich das Maul zerreißen / olle Kammel / Klappe halten / ihr könnt mich mal / das kann nicht sein.

\section{Fazit und Ausblick}

Zusammenfassend lässt sich feststellen, dass sich das untersuchte Interview mit Boris Becker unter dem sprachlich-pragmatischen Aspekt als besonders ergiebig erweist. Dies betrifft vor allem die sprachliche Seite - es wurde nachgewiesen, dass das Interview viele emotionale Mittel enthält, nicht nur Phraseologismen, sondern auch einige gesprochensprachliche Formeln und Ausdrücke sowie einen umgangssprachlichen Wortschatz. Diese Mittel und ihr expressiver Charakter erwecken Emotionen sowohl bei dem Interviewten als auch beim Leser. Durch ihren Einsatz wird die in der Unterhaltung des Lesers liegende Funktion der Interviews mit Prominenten erfüllt. Der Leser hat den Eindruck, er stehe dem Interviewten näher, er hat die Möglichkeit, einiges aus den privaten Angelegenheiten einer prominenten Person zu erfahren. Des Weiteren darf man nicht vergessen, dass das durchgeführte Interview der Selbstdarstellung des Befragten dient. Das Interview gibt ihm einen großen Spielraum für die Darstellung aller seiner Selbstbilder - er möchte den Ruf eines hervorragenden Sportlers, anständigen Vaters und erfolgreichen Geschäftsmanns haben. Die oben genannten Strategien (widersprechen, vorwerfen, zurückweisen) und sprachliche Mittel ihrer Realisierung beweisen die große Emotionalität des Gespräches und machen es somit für den Leser besonders spannend. 
Vor dem Hintergrund des face-Konzepts und der Image-Arbeit kommt diesen Strategien und den dargestellten Selbstbildern eine wichtige Funktion zu - sie sollen das positive Gesicht des Interviewten wahren, aber auch das negative Gesicht, indem seine Handlungsfreiheit gewährleistet und gewisse Autonomie und Individualität unterstützt werden.

Die Beschäftigung mit diesem Interview und mit Presseinterviews allgemein lassen einige Desiderate erkennen. So werden Phraseologismen, die hier eine bedeutsame Rolle spielen, in der Fachliteratur anhand verschiedener Pressetextsorten ermittelt, aber selten in Interviews. Es steht dabei außer Zweifel, dass gerade meinungsbetonte Texte, zu denen Interviews zählen, ,reich an Phraseologismen sind“"(Skod-Södersved 2007: 273). Das zweite Gebiet betrifft die Grenze zwischen Mündlichkeit und Schriftlichkeit in solchen Texten. Es wurden viele emotionale und umgangssprachliche Ausdrücke ermittelt sowie gesprochensprachliche Formeln, die für die Mündlichkeit und kommunikative Nähe charakteristisch sind, die hier aber in einem Schrifttext erscheinen. Und - last but not least - Interviews sind eine Interaktion mindestens zweier Menschen, die einen großen Spielraum für reaktive und nicht-reaktive Handlungen lassen. Auch in diesem Bereich zeigt sich ein großes Untersuchungspotenzial.

\section{Literatur}

\section{Quelle}

Der Spiegel 34/2014: „Ich bin der Drachentöter“ - Interview mit Boris Becker.

\section{Sekundärliteratur}

Brown, Penelope / Levinson, Stephen C. (1987): Politeness. Some universals in language usage. Cambridge.

Fiehler, Reinhard (2008): Emotionale Kommunikation. In: Fix, Ulla / Gardt, Andreas / Knape, Joachim (Hrsg.): Rhetorik und Stilistik. Ein internationales Handbuch historischer und systematischer Forschung. Halbband 1. Berlin/New York. S. 757-772.

Holly, Werner (2001): Beziehungsmanagement und Imagearbeit. In: Brinker, Klaus / Antos, Gerd / Heinemann, Wolfgang / Sager, Sven F. (Hrsg.): Text- und Gesprächslinguistik. Halbband 2. Berlin/New York. S. 1382-1393.

Lüger, Heinz-Helmut (2014): Meledicere et delectare? Unhöflichkeit als Persuasions- und Unterhaltungsstrategie. In: Lenk, Hartmut E.H. / Suomela-Härmä, Elina (Hrsg.): Höflichkeit in Textsorten. Landau. S. 41-62.

Schwitalla, Johannes (1979): Dialogsteuerung in Interviews. Ansätze zu einer Theorie der Dialogsteuerung mit empirischen Untersuchungen von Politiker-, Experten- und Starinterviews in Rundfunk und Fernsehen. München. 
Schwitalla, Johannes (1996): Beziehungsdynamik. Kategorien für die Beschreibung der Beziehungsgestaltung sowie der Selbst- und Fremddarstellung in einem Streit- und Schlichtungsgespräch. In: Kallmeyer, Werner (Hrsg.): Gesprächsrhetorik. Rhetorische Verfahren im Gesprächsprozeß. Tübingen. S. 279-349.

Skog-Södersved, Mariann (2007): Phraseologismen in den Printmedien. In: Burger, Harald / Dobrovol'skij, Dmitrij / Kühn, Peter / Nordisk, Neal R. (Hrsg.): Phraseologie. Ein internationales Handbuch der zeitgenössischen Forschung. Halbband 1. Berlin. S. 269-275.

Germanica Wratislaviensia 143, 2018

(C) for this edition by CNS 